\title{
Determinação e análise de parâmetros para 0 desenvolvimento de um processo de decoração de peças plásticas através da injeção sobre tecido
}

\section{Determination and analysis of parameters for the development of a process for decoration plastic parts by injection on fabrics}

\author{
Cristiano Linck $^{1 *}$, Carmen lara Walter Calcagno ${ }^{1}$ e João Antônio Pinto de Oliveira ${ }^{1}$ \\ ${ }^{1}$ Instituto Federal de Educação, Ciência e Tecnologia Sul-rio-grandense - IFSUL, \\ Campus Sapucaia do Sul, Sapucaia do Sul, RS, Brasil \\ *cristianol@sapucaia.ifsul.edu.br; cristiano.linck@senairs.org.br
}

\begin{abstract}
Resumo
O processo de injeção sobre tecidos, utilizado na indústria automotiva, é caracterizado por modificações no processo de injeção convencional e possibilita o desenvolvimento de novos produtos com características diferenciadas. Devido às dificuldades de processamento, e tendo em vista as necessidades de adaptação dos parâmetros de injeção quando essa é feita sobre tecidos, foi desenvolvida uma metodologia de determinação e análise de parâmetros de processo que possibilitaram o desenvolvimento desta técnica. Os testes foram realizados inicialmente em uma indústria produtora de peças automotivas e a metodologia adotada foi repetida em uma instituição de ensino. Verificou-se, então, que controlando os parâmetros de injeção e fechamento do molde, foi possível obter, em ambos os casos, peças conforme as especificações de qualidade requeridas.
\end{abstract}

Palavras-chave: injeção, processos não convencionais, IMD, injeção sobre tecido, parâmetros de injeção, desenvolvimento de processos.

\section{Abstract}

The injection molding process on fabrics used in the automotive industry is characterized by changes in conventional injection molding process and enables the development of new products with different characteristics. Due to processing difficulties, and considering the need for adaptation of injection parameters when it is done on fabrics, a method for determining and analyzing the process parameters was developed so that technique could be put into practice. Initially tests were conducted in an industry producing automotive parts and the method adopted was replicated in an educational institution. Then, it has been found, that through controlling the injection and mold closing parameters, it was possible to obtain, parts conforming to quality specifications required in both cases.

Keywords: injection, unconventional processes, IMD, injection on fabrics, injection molding parameters, process development.

\section{Introdução}

Os artefatos plásticos constituem uma grande parcela da produção de bens de consumo mundiais. As exigências de design e aparência pelos consumidores se tornou algo indispensável à sobrevivência das empresas que produzem estes artefatos.

Com o passar dos anos processos não convencionais de injeção têm sido constantemente desenvolvidos. Um exemplo é a injeção sobre tecido, conhecida como IMD (in mold decoration) ou backmolding, que visa à produção de peças plásticas com um revestimento de tecido durante apenas uma etapa de produção ${ }^{[1]}$.

O processo convencional de produção de peças revestidas com tecido é realizado em duas etapas, a injeção e a colagem do revestimento. Este processo apresenta desvantagens como a geração de bolhas, o descolamento dos materiais e existem custos associados às etapas de pós-produção.

Os processos de injeção não convencionais são, em grande parte, semelhantes ao processo convencional. Todos 
possuem as fases de fechamento do molde, dosagem da matéria-prima, injeção, pressurização, recalque, abertura do molde, extração da peça e tempo de pausa. Eles se diferenciam apenas em etapas complementares que podem ocorrer antes, durante ou depois da injeção propriamente dita. Essas diferenciações proporcionam a obtenção de produtos inovadores com características diferentes que são dificilmente alcançadas por processos convencionais de injeção ${ }^{[2,3]}$.

O processo de injeção de plástico pode produzir peças de várias cores e também com superfícies texturizadas. Contudo, frequentemente, o acabamento não satisfaz a todos os requisitos do projeto e, consequentemente, dos clientes, como por exemplo, a sensação produzida pelo contato (tato) proporcionada por uma peça revestida com tecido ${ }^{[1,4]}$. A técnica de moldagem sobre tecidos, filmes ou lâminas de outros materiais é realizada através de uma sobre injeção e teve início na década de 1980, mas somente uma década depois, a partir de 1990 foi desenvolvida em sua plenitude ${ }^{[5]}$.

Nos últimos anos a tecnologia IMD ganhou atenção do setor automotivo, tendo em vista, por exemplo, a redução de custos e problemas no produto associados à colagem da camada decorativa sobre a peça. Em contrapartida, o IMD se torna extremamente caro quando se utilizam linhas inteiras de robôs manipuladores para colocar, retirar, rebarbar o excesso das camadas decorativas, além de transportar e finalizar o processo produtivo das peças ${ }^{[5,6]}$.

Alguns problemas são comuns no IMD, como a migração de material através da camada decorativa, o rasgamento do tecido por excesso de força de fechamento e o enrugamento do tecido por causa do fluxo de material polimérico $^{[1]}$.

A pressão dentro da cavidade deve ser a menor possível no IMD para evitar danos ao tecido durante a injeção ou a migração do material polimérico através dele. A minimização da pressão favorece um preenchimento mais suave da peça e, além de prevenir marcas de fluxo ou diferenças de espessura da parede da peça, evita que o tecido seja danificado ou deformado durante a injeção, bem como, evita a compactação excessiva do material durante a injeção ${ }^{[1,7]}$.

Outro fator importante de ser considerado é que, por se tratar de um material decorativo extremamente sensível, o tecido pode entrar em colapso caso a tensão de compressão proporcionada pelo fechamento do molde seja demasiadamente elevada, causando o rasgamento do tecido principalmente em regiões em que há cantos vivos ${ }^{[4]}$. Para evitar esse tipo de problema é necessário, além de reduzir a pressão de fechamento do molde, deixar a altura de molde maior proporcionando um fechamento mais suave do mesmo ${ }^{[1]}$.

Nesse trabalho tem-se como objetivo determinar os parâmetros de injeção sobre tecidos utilizando-se máquinas e moldes diferentes. Com os resultados pretende-se mensurar a variação em relação ao convencional e replicar as estratégias em equipamentos e moldes diferentes.

\section{Experimental / Métodos e Materiais}

Os parâmetros iniciais de processo foram obtidos através da produção de peças utilizando o processo de injeção convencional. Posteriormente, as peças foram produzidas inserindo o tecido no molde, sendo realizados ajustes nos parâmetros de processo para a obtenção de produtos conforme as especificações dos clientes. O estudo foi realizado em duas etapas: uma na Pelzer Sistemas do Brasil e outra no Instituto Federal Sul-rio-grandense Campus Sapucaia do Sul (IFSul). Na Pelzer Sistemas do Brasil foi utilizada a injetora Haitian 2000 MA com capacidade de injeção de $334 \mathrm{~cm}^{3}$ e 200 toneladas de força de fechamento, um molde para descanso de braço (Arm Rest), o Material Polimérico PP-T2 Jet Black 600R (GMWP6208) fornecido pela Styron e os Tecidos Mana Very Dark de coloração cinza clara e o Mana Jet Black de coloração cinza escura ambos com o desenvolvimento patenteado pela General Motors. No IFSul foi utilizada a Máquina Romi Primax 150 com força de fechamento de 150 toneladas e capacidade de plastificação de $350 \mathrm{~cm}^{3}$, um molde para corpos-de-prova, o Material Polimérico PP H107 da Braskem e o tecido sendo o mesmo utilizado na Pelzer Sistemas do Brasil.

\section{Resultados e Discussões}

\subsection{Determinação dos parâmetros na Pelzer Sistemas do Brasil}

Nos testes realizados na empresa Pelzer Sistemas do Brasil analisou-se cada etapa do processo e foram estabelecidos parâmetros que possibilitaram o desenvolvimento deste processo novo e, consequentemente, a produção dessas peças em larga escala para atender a demanda da linha da General Motors.

O molde do Descanso de Braço, utilizado nesse estudo, foi desenvolvido com o propósito de utilizá-lo em processo IMD. Esse molde, cujo projeto é complexo e pensado no propósito do IMD, possui câmara quente, acionamento de machos hidráulicos, tem pinos e alojamentos específicos para a inserção do tecido, além de uma estrutura de fechamento suavizada, sem cantos vivos e reentrâncias para evitar o rasgamento do tecido durante o fechamento do molde. O molde produz a peça, visualizada na Figura 1, que faz parte da porta dos carros da linha Onix para apoiar o braço, o descanso de braço.

\subsection{Determinação inicial de parâmetros}

A dosagem, o perfil de abertura do molde e o perfil de extração da peça são parâmetros que não variam com a inserção do tecido no processo e foram determinados baseados na metodologia primária da injeção convencional. Estes parâmetros permaneceram constantes durante todos os testes realizados.

As temperaturas foram definidas conforme recomendação do fornecedor para o material, sendo utilizado um perfil decrescente do bico de injeção para 
a garganta de alimentação, sendo elas, respectivamente, $220^{\circ} \mathrm{C}, 210^{\circ} \mathrm{C}, 205^{\circ} \mathrm{C}, 200^{\circ} \mathrm{C}, 185^{\circ} \mathrm{C}$.

Tendo em vista o conhecimento prévio da influência da pressão na cavidade e da pressão de fechamento no desempenho do IMD, utilizou-se uma programação da injetora que iniciou a etapa de injeção com velocidades e pressões $80 \%$ mais baixas do que no processo convencional. Além disso, optou-se também por usar duas fases de injeção, sendo a segunda ligeiramente mais rápida e com maior pressão de injeção.

O fechamento do molde foi programado utilizando-se um perfil de quatro fases e, semelhante à etapa de injeção, foram utilizadas pressões e velocidades baixas, especialmente nas últimas fases de fechamento. $\mathrm{O}$ maior valor de pressão de fechamento utilizado é equivalente a $25 \%$ da capacidade de fechamento da máquina injetora (200 bar) e 27,8\% menor do que o necessário previamente estimado com base no peso da peça e área projetada do molde para injetar a peça sem tecido, ou seja, 180 bar.
Além disso, a altura de molde utilizada foi $4 \mathrm{~mm}$ maior (espessura do tecido), evitando o rasgamento do tecido em decorrência de um travamento muito justo.

As ações preventivas na programação do fechamento alcançaram os objetivos pretendidos e não houve rasgamento ou enrugamento do tecido durante os testes.

\subsection{Defeitos nas peças injetadas}

Embora os parâmetros pré-definidos tenha evitado o rasgamento e enrugamento do tecido, durante a primeira avaliação no processo de injeção surgiram dois problemas que já eram previstos na literatura específica. O primeiro deles foi a migração de material polimérico através do tecido, associado a um excesso de compactação, que deve ter sido promovida pelo uso de pressões e velocidades de injeção superiores a resistência do tecido. O problema da migração de material sobre o tecido pode ser observado na Figura 2a e, com o intuito de eliminar este problema, as

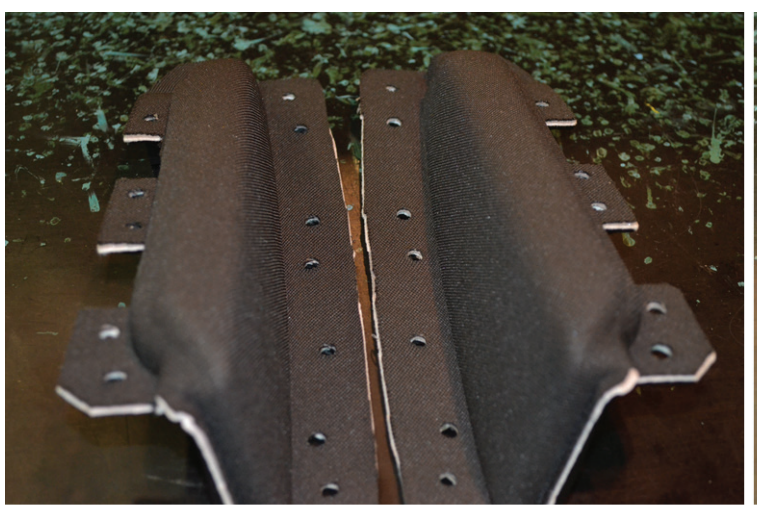

(a)

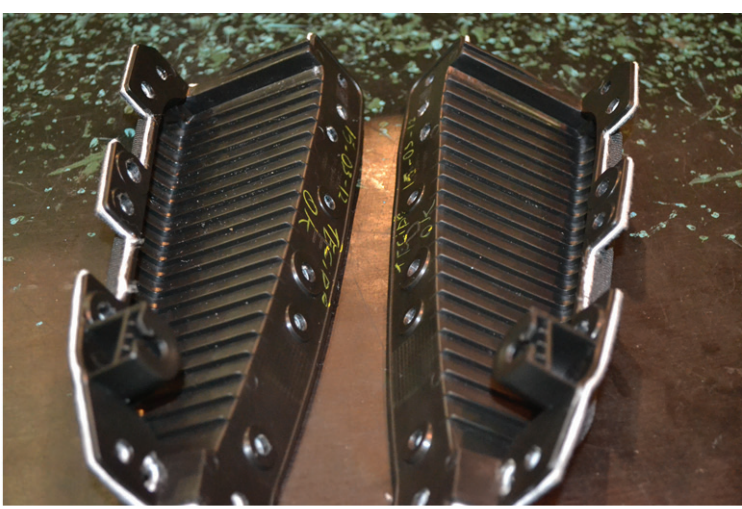

(b)

Figura 1. Peças Arm Rest rebarbadas (a) lado visível; (b) lado não visível.

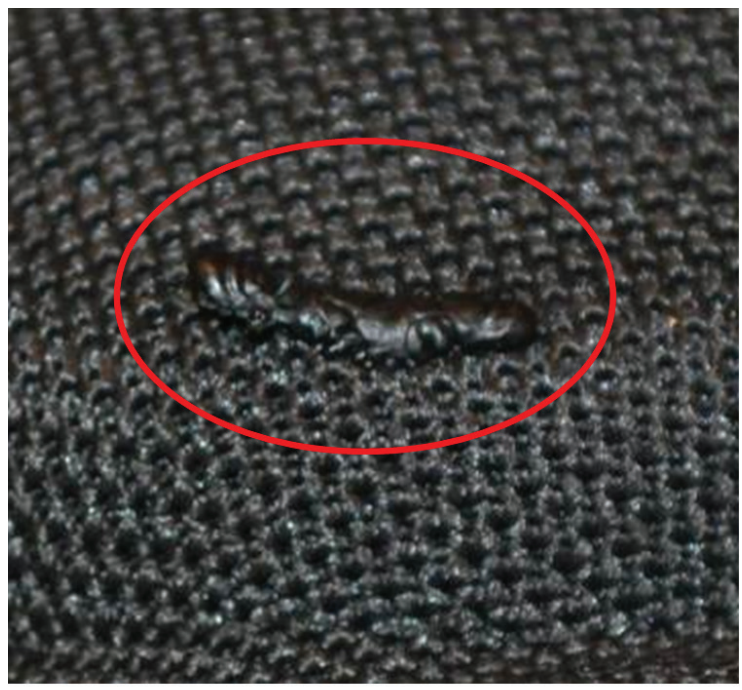

(a)

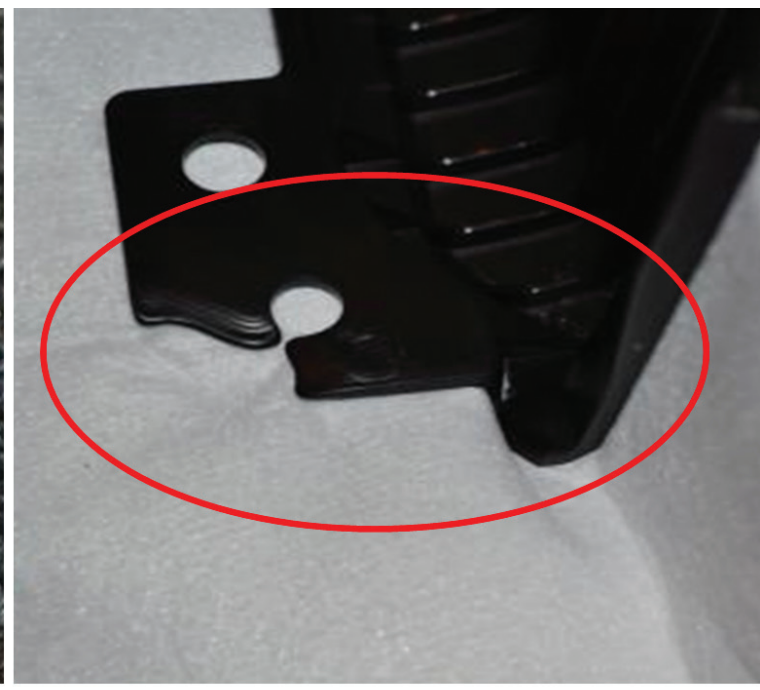

(b)

Figura 2. a) defeito de migração de material polimérico através do tecido; b) falta de preenchimento. 
velocidades e pressões foram reduzidas gradativamente até que uma peça isenta do problema fosse obtida.

Contudo, com a redução das velocidades e pressões de injeção foi percebido que não houve o preenchimento completo do lado direito da peça, conforme é mostrado na Figura 2b. Esta situação é normal no processo de injeção, sendo que há várias maneiras comuns de solucioná-la, como por exemplo, utilizar pressões de injeção maiores (solução mais comum). Porém, na injeção sobre tecido, essa não seria uma solução viável, tendo em vista a migração de material. Portanto esta ação rotineira não possibilitou a eliminação do problema e a solução encontrada nesse caso específico será apresentada a seguir.

\subsection{Determinação final dos parâmetros}

Antes de avaliar novas estratégias de injeção, fez-se a opção de analisar o molde quanto ao balanceamento das cavidades e foi verificado que havia desbalanceamento dos canais. Essa situação pode promover, em situações extremas, a ocorrência de duas situações antagônicas. Por um lado, pode ocorrer a falha no preenchimento em determinada região da cavidade enquanto que, em outra região da peça, pode ocorrer compactação excessiva. A migração de material pelo tecido pode ser decorrente da compactação excessiva ocasionada pela busca de condições de preenchimento total da peça. Essa situação é percebida claramente no IMD e não na injeção convencional.

No molde em estudo é observada a compactação preferencial em uma das cavidades. Esta situação se deve a entrada de maior quantidade de material na cavidade de fluxo facilitado para permitir o completo preenchimento da cavidade onde o fluxo é difícil. Com o intuito de promover um preenchimento mais uniforme, de ambas as cavidades, os canais foram balanceados.

As temperaturas estabelecidas no primeiro teste revelaram-se excessivas para injeção sobre tecido. Optou-se por baixar as temperaturas a fim de aumentar a viscosidade polimérica e consequentemente evitar a compactação excessiva das peças. As temperaturas foram determinadas reduzindo-se $10^{\circ} \mathrm{C}$ por zona de aquecimento.
Por fim, a injeção foi programada com duas fases de preenchimento, seguindo a mesma estratégia incialmente definida. Mesmo com os canais balanceados, o lado direito continuava falhando enquanto o esquerdo completava e apresentava, consequentemente, migração do material polimérico através do tecido. Neste momento foi decidido avaliar a influência dos comprimentos dos três canais de distribuição que preenchem as cavidades e percebeu-se que as distâncias aumentaram gradativamente do topo da peça para a parte inferior da mesma, conforme Figura 3a.

Após a análise das distâncias dos canais foi decidido acrescentar uma terceira fase de injeção a fim de compensar a diferença de comprimento do fluxo.

Assim foi possível determinar uma programação que atendesse aos requisitos técnicos da peça com repetibilidade e sem a apresentação dos defeitos que estavam ocorrendo anteriormente. A programação da injeção pode ser visualizada na Tabela 1. Como resultado final da injeção foram obtidas peças isentas de falhas, sem rugas no tecido ou passagem do material termoplástico para o outro lado do mesmo.

\subsection{Determinação de parâmetros no Instituto Federal Sul-rio-grandense}

\subsubsection{Modificações na ferramenta}

Tendo em vista os conhecimentos adquiridos nos testes realizados na produção do "descanso de braço", o molde definido para estudo no IFSul foi avaliado quanto a geometria das cavidades. Após a análise foi possível concluir que este não possuía excessos de cantos vivos e reentrâncias que pudessem comprometer o tecido. Também foi analisado que o tecido poderia ser fixado nas próprias colunas-guia que alinham o fechamento da placa móvel com a placa fixa do molde.

Por se tratar de um molde família, ou seja, com múltiplas cavidades diferentes, verificou-se um desbalanceamento no mesmo, que poderia resultar em um fluxo desuniforme e, consequentemente, o preenchimento não homogêneo de todas as cavidades: umas preencheriam antes do que as outras. Na Figura 3b são mostradas as diferentes

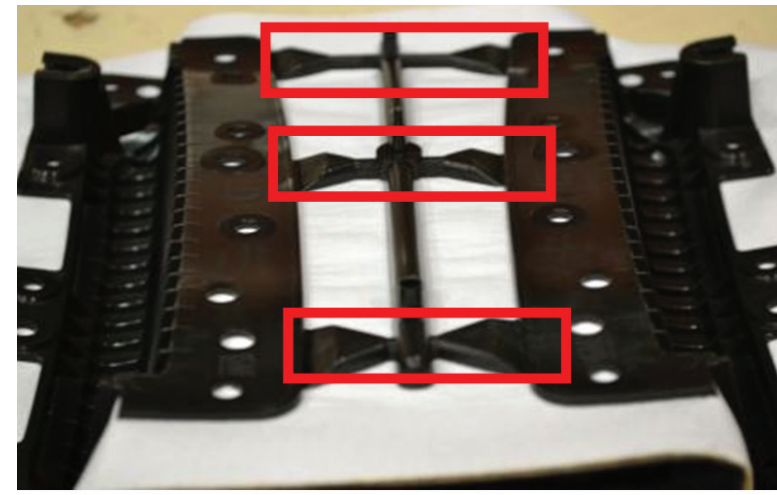

(a)

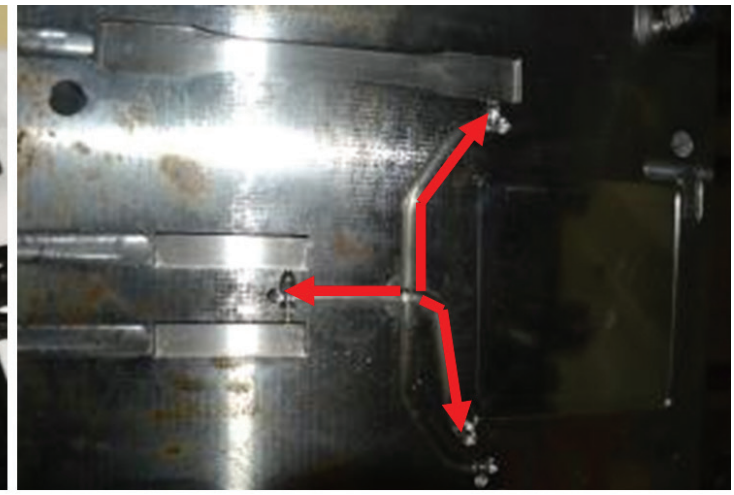

(b)

Figura 3. a) comprimento dos canais de injeção do Arm Rest; b) comprimento dos canais de alimentação e distribuição do molde dos corpos-de-prova. 
cavidades produzidas pelo mesmo molde e a diferença do comprimento dos canais de distribuição e alimentação destas cavidades. Esta diferença de comprimento dos canais tem o mesmo impacto no processo que os observados no molde do descanso de braço (Figura 3a). Conforme os resultados obtidos na etapa anterior, sabe-se que este fato influencia diretamente nos resultados do IMD.

\subsubsection{Modificações no processo (Ajuste de Parâmetros)}

Inicialmente foram injetadas as peças sem o tecido para obter-se uma avaliação sobre como ocorria o preenchimento do molde.

\subsubsection{Parâmetros de injeção}

Seguindo a mesma metodologia aplicada na empresa Pelzer Sistemas do Brasil, inicialmente foram determinados parâmetros para o processo na ausência do tecido. Os parâmetros que não variam com a inserção do tecido foram os mesmos para ambos os testes.

Foram definidas temperaturas diferentes, ou seja, $200^{\circ} \mathrm{C}, 190^{\circ} \mathrm{C}, 180^{\circ} \mathrm{Ce} 160^{\circ} \mathrm{C}$, do bico de injeção à garganta de alimentação, devido a troca do material polimérico.

$O$ fechamento do molde foi determinado com um perfil de cinco fases com pressões e velocidades bastante baixas, especialmente nas últimas fases de fechamento para que não houvesse rasgamento do tecido, como pode ser observado na Tabela 2.

Para possibilitar a injeção sobre tecido foi ajustada a altura de molde de modo que o fechamento do molde ocorresse no tecido e não nas partes metálicas do molde, evitando problemas previstos anteriormente na empresa. Após ser inserido o tecido, a injeção foi realizada com valores de velocidade e pressão baixas e com um tempo de injeção alto para proporcionar um preenchimento completo da peça utilizando a dosagem pré-estipulada para a injeção sem o tecido.

O primeiro resultado dos parâmetros determinados foi satisfatório possibilitando a injeção da cavidade quadrada perfeitamente. As outras cavidades, porém, apresentaram uma forte migração do material polimérico através do tecido devido ao desbalanceamento dos canais de distribuição do molde. Este problema foi previsto previamente analisando a diferença de tamanho das cavidades e canais.

A Figura 4a mostra a parte visível da injeção de todas as cavidades onde a cavidade quadrada foi obtida sem falhas de preenchimento, rugas ou rasgamento do tecido e sem a migração do material polimérico para o lado visível. E a Figura $4 \mathrm{~b}$ mostra a parte não visível das peças injetadas sobre tecido. É possível perceber o efeito da migração do material nas demais cavidades pelo escurecimento das pontas das mesmas, conforme destacado na imagem.

$\mathrm{Na}$ Figura 4c é possível visualizar a parte visível da peça obtida no segundo teste, já rebarbada com uma tesoura, comprovando a qualidade esperada para uma peça decorada com tecido, sem rugas, falhas ou migração de material. É importante ressaltar que as imperfeições das bordas da peça são devido à má retirada do excesso do tecido da mesma. $\mathrm{Na}$ Figura $4 \mathrm{~d}$ é possível observar a parte não visível da peça

Tabela 1. Perfil de fechamento e injeção.

\begin{tabular}{lcccc}
\hline & \multicolumn{1}{c}{ FECHAMENTO } \\
\hline \multicolumn{1}{c}{ ETAPA } & $\mathbf{1}$ & $\mathbf{2}$ & BAIXA PRESSÃO & ALTA PRESSÃO \\
\hline POSIÇÃO (mm) & 350 & 120 & 10 & 0 \\
PRESSÃO (bar) & 20 & 20 & 15 & 50 \\
VELOCIDADE & 5 & 10 & 7 & 10 \\
\hline \multicolumn{1}{c}{ ETAPA } & & INJEÇÃO & $\mathbf{2}$ & 1 \\
\hline POSIÇÃO (mm) & $\mathbf{3}$ & & 100 & 160 \\
PRESSÃO (bar) & 45 & 40 & 15 \\
VELOCIDADE (\%) & 20 & & 40 & 10 \\
\hline
\end{tabular}

Tabela 2. Perfil de fechamento e injeção.

\begin{tabular}{|c|c|c|c|c|c|c|c|c|c|c|c|}
\hline \multicolumn{12}{|c|}{ FECHAMENTO } \\
\hline ETAPA & \multicolumn{2}{|l|}{1} & \multicolumn{2}{|l|}{2} & \multicolumn{2}{|r|}{3} & \multicolumn{4}{|c|}{ Baixa Pressão } & Alta Pressão \\
\hline POSIÇÃO (mm) & \multicolumn{2}{|l|}{600} & \multicolumn{2}{|l|}{400} & \multicolumn{2}{|r|}{375} & \multicolumn{4}{|c|}{275} & 21 \\
\hline PRESSÃO (bar) & \multicolumn{2}{|l|}{50} & \multicolumn{2}{|l|}{45} & \multicolumn{2}{|r|}{25} & \multicolumn{4}{|c|}{15} & 60 \\
\hline VELOCIDADE (\%) & \multicolumn{2}{|l|}{30} & \multicolumn{2}{|l|}{50} & \multicolumn{2}{|r|}{40} & \multicolumn{4}{|c|}{10} & 100 \\
\hline \multicolumn{12}{|c|}{ INJEÇÃO } \\
\hline ETAPA & FINAL & 9 & 8 & 7 & 6 & 5 & 4 & 3 & 2 & 1 & INÍCIO \\
\hline POSIÇÃO (mm) & 6 & 8 & 10 & 13 & 15 & 18 & 20 & 22 & 25 & 27 & 30 \\
\hline PRESSÃO (bar) & \multicolumn{11}{|c|}{50} \\
\hline VELOCIDADE (\%) & & 19 & 48 & 87 & 106 & 67 & 48 & 22 & 9 & 9 & 9 \\
\hline \multicolumn{12}{|c|}{ RECALQUE } \\
\hline TEMPO (s) & 0,3 & 0,6 & 0,9 & 1,2 & 1,5 & 1,8 & 2,1 & 2,4 & 2,7 & 3,0 & \\
\hline PRESSÃO (bar) & & & & & & 30 & & & & & \\
\hline
\end{tabular}




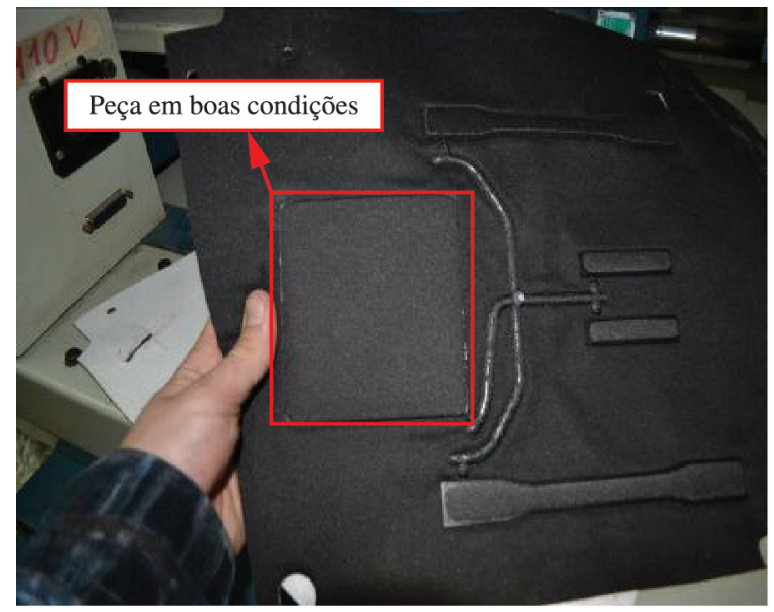

(a)

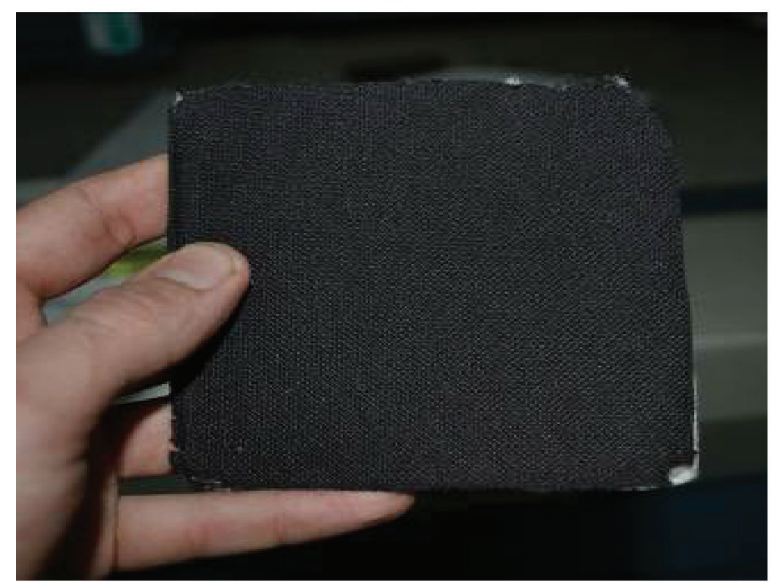

(c)

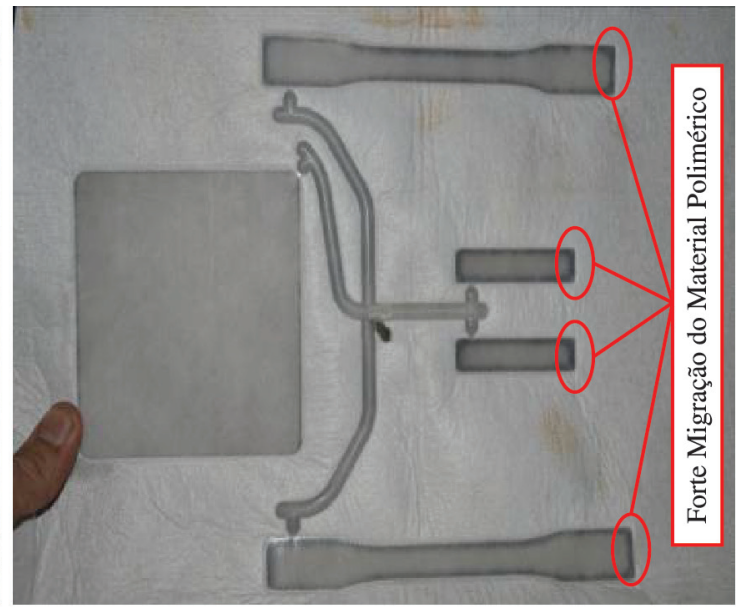

(b)

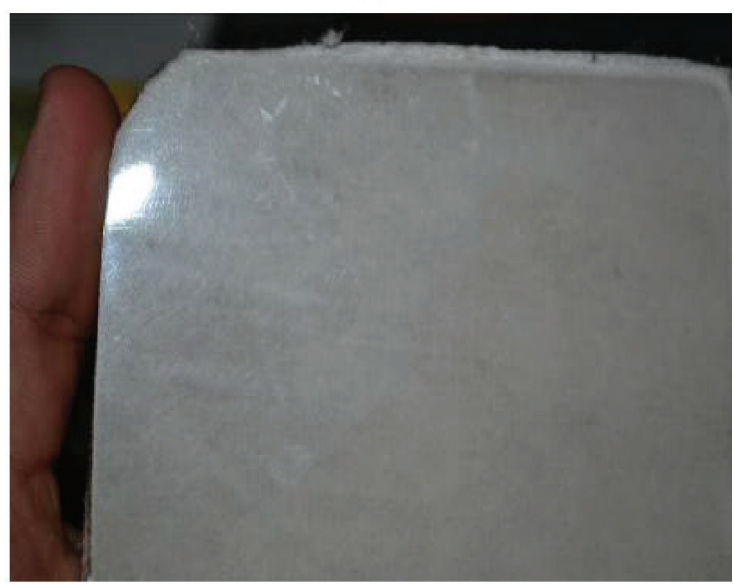

(d)

Figura 4. a) parte não visível da cavidade quadrada após a retirada do excesso de tecido; b) parte não-visível; c) parte visível da injeção sobre tecido no IFSul; d) parte não-visível. Detalhe na migração do material polimérico através do tecido.

obtida no segundo teste, rebarbada com uma tesoura, na qual se observa o padrão de qualidade esperado para uma peça decorada com tecido.

\section{Conclusões}

Baseado na aplicação dos conhecimentos adquiridos através da revisão de bibliografia foi possível elaborar uma estratégia que possibilitou o desenvolvimento do processo de decoração de peças plásticas através da injeção sobre tecido na empresa Pelzer Sistemas do Brasil, no Complexo Automotivo da General Motors em Gravataí, Rio Grande do Sul.

Após desenvolver este processo, foi escolhido um molde para ser injetado com um material diferente em uma injetora disponível no Instituto Federal de Educação, Ciência e Tecnologia Sul-rio-grandense (IFSul), Campus Sapucaia do Sul, Rio Grande do Sul. E baseado mais uma vez em uma estratégia e análise bem estruturadas foi possível desenvolver novamente o processo.
Comprovou-se que a utilização de baixas pressões e velocidades de injeção é indispensável para a injeção sobre tecido, uma vez que pressões e velocidades elevadas provocam a migração do material polimérico através do tecido ou enrugamento do tecido durante a injeção. Contudo, pressões e velocidades demasiadamente baixas podem provocar, em contrapartida, falhas de preenchimento.

Comprovou-se também que baixas pressões na fase de fechamento, em especial, próximas ao travamento do conjunto de fechamento, garantem a integridade do tecido. Quanto ao fechamento é importante ressaltar, ainda, que um alívio da altura de molde se faz necessário, pois o tecido no centro do molde impede o travamento que ocorreria caso o mesmo não estivesse ali. Também é possível utilizar o alívio da altura de molde para garantir maior integridade do tecido evitando rasgos ou amassamentos do mesmo.

Verificou-se que o balanceamento dos canais de injeção é de fundamental importância para o bom desenvolvimento do processo de injeção sobre tecido, pois evita a compactação excessiva de material polimérico na cavidade em que o fluxo é facilitado. Esta compactação 
excessiva pode causar o defeito de migração de material polimérico através do tecido.

Assim foi demonstrado que o processo de injeção sobre tecido pode ser desenvolvido com diferentes moldes, materiais poliméricos e máquinas, variando os parâmetros de processo para obter peças isentas de defeitos, seguindo a estratégia pré-definida com embasamento teórico.

\section{Referências}

1. Fischbach, G. (1994). US Patent No 5.356.576: Process for manufacture of plastics moldings with decorative coating. Washington: U.S. Patent and Trademark Office.

2. Manrich, S. (2005). Processamento de termoplásticos: rosca única, extrusão e matrizes, injeção e moldes. São Paulo: Artliber.

3. Engel. (2012). Catálogo da Engel: Engel Application Technology: Innovate injection moulding techology for special products. Recuperado em http://www.lakara.si/wp-content/ uploads/2012/09/Aplication-technology-EN.pdf
4. Gonçalves, M. A. (2014, Setembro 25). Utilização de sistemas valvulados sequenciais para decoração de peças técnicas. Recuperado em http://www.moldesinjecaoplasticos.com.br/ sistemasvalvulados.asp

5. Renners, C. (2010). Soluções sistêmicas para células de produção complexas - Tendências modernas na decoração "In Mold". In Anais do Simpósio Internacional de Injeção de Plásticos 2010: "Otimização de Recursos Produtivos" (pp. 67-89). São Paulo: Instituto Nacional do Plástico.

6. Battenfeld. (2012). Catálogo da Battenfeld: Back Moulding Technology: Decorated parts in one process step. Recuperado em http://www.battenfeld.ru/fileadmin/templates/docs/ technologies/back\%20moulding\%20technology.pdf

7. Vasilj, A., Schmidt, M., \& Mitzler, J. (2003). In-mould decoration on modified standard machines. Kunststoffe Plast Europe, 3, 65-67.

Enviado: Mar: 12, 2014

Reenviado: Set. 19, 2014 Aceito: Out. 10, 2014 